\title{
Placental Finding
}

National Cancer Institute

\section{Source}

National Cancer Institute. Placental Finding. NCI Thesaurus. Code C117337.

Clinical and laboratory findings about the placenta. 